\title{
Occlusion pressure analysis role in partitioning of pulmonary vascular resistance in CTEPH
}

\author{
Mark Toshner*\#, Jay Suntharalingam", Pierre Fesler ${ }^{+}$, Elaine Soon*, \\ Karen K. Sheares" ${ }^{\#}$, David Jenkins" ${ }^{\#}$ Paul White ${ }^{\S}$, Nicholas W. Morrell*, \\ Robert Naeije ${ }^{f}$ and Joanna Pepke-Zaba ${ }^{\#}$
}

ABSTRACT: Flow-directed pulmonary artery occlusion is posited to enable partitioning of vascular resistance into small and large vessels. As such it may have a role in assessment for pulmonary endarterectomy.

To test if the occlusion technique distinguished small from large vessel disease we studied $\mathbf{5 9}$ subjects with chronic thromboembolic pulmonary hypertension (CTEPH), idiopathic pulmonary arterial hypertension (IPAH), and connective tissue disease (CTD)-associated PAH. At right heart catheterisation, occlusion pressures were recorded. With fitting of the pressure decay curve, pulmonary vascular resistance was partitioned into downstream (small vessels) and upstream (large vessels, Rup).

47 patients completed the study; 14 operable CTEPH, 15 inoperable CTEPH, 13 idiopathic or CTD-PAH and five post-operative CTEPH. There was a significant difference in mean Rup in the proximal operable CTEPH group 87.3 (95\% Cl 84.1-90.5); inoperable CTEPH mean 75.8 (95\% Cl 66.76-84.73), $p=0.048$; and IPAH/CTD, mean 77.1 (95\% Cl 71.86-82.33), $p=0.003$. Receiver operating characteristic curves to distinguish operable from inoperable CTEPH demonstrated an area under the curve of $0.75, p=0.0001$. A cut-off of 79.3 gave $100 \%$ sensitivity $(95 \% \mathrm{Cl} 73.5-100 \%)$ but $57.1 \%$ specificity $(95 \% \mathrm{Cl} 28.9-82.3 \%)$. In a subgroup analysis of multiple lobar sampling there was demonstrable heterogeneity.

Rup is significantly increased in operable proximal CTEPH compared with non-operable distal CTEPH and IPAH/CTD-PAH. Rup variability in patients with CTEPH and PAH is suggestive of pathophysiological heterogeneity.

KEYWORDS: Pulmonary endarterectomy, pulmonary hypertension, thrombosis

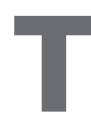
he pulmonary artery occlusion technique has emerged as a possible way of partitioning vascular resistance across the pulmonary vascular bed. This is of particular relevance in chronic thromboembolic pulmonary hypertension (CTEPH). In CTEPH the ability to discriminate the contribution of small vessel vasculopathy from organised thrombus in large vessels is critical to treatment decisions. The gold standard treatment of choice is pulmonary endarterectomy (PEA) surgery. Distal disease, whether vasculopathic or small vessel thrombotic, is a major risk factor for poorer outcome [1-3]. It has, however, been demonstrated that patients with high pulmonary vascular resistance (PVR) can still potentially benefit from operative intervention. THISTLETHWAITE et al. [4] have reported a greater reduction in systolic pulmonary arterial pressure and PVR in a cohort of patients with a very high mean PVR of $1,299 \mathrm{dyn} \cdot \mathrm{s} \cdot \mathrm{cm}^{-5}$ when compared to a cohort of more modest PVR ( $\left.832.5 \mathrm{dyn} \cdot \mathrm{s} \cdot \mathrm{cm}^{-5}\right)$, although the high PVR group had a lower survival $(89.2 \%$ versus $96.5 \%)$. Additional discriminators to identify those at risk of poor outcome are therefore desirable. In this context, KIM et al. [5] have previously reported results from a cohort of patients undergoing PEA that suggest the occlusion technique is able to effectively partition resistance into small and large vessel compartments. The occlusion derived partition of resistance correlated strongly with
AFFILIATIONS

*University of Cambridge,

\#Papworth Hospital,

${ }^{\S}$ Addenbrooke's Hospital, Cambridge,

"Royal United Hospital, Bath, UK. +Hôpital Lapeyronie, Montpellier, France.

${ }^{\text {fH}}$ Hôpital Erasme, Brussels, Belgium.

CORRESPONDENCE

J. Pepke-Zaba

Papworth Hospital

Papworth Everard

Cambridge

CB23 3RE

UK

E-mail: Joanna.Pepkezaba@

papworth.nhs.uk

Received:

Aug 052011

Accepted after revision:

Jan 172012

First published online:

Feb 232012 
immediate post-operative results and additionally demonstrated promise in identifying those at high operative risk regardless of PVR.

There are issues related to the validity of the technique in this context. There are questions about the size of vessel that partitioning segregates, and it is possible that it does not properly assess the contribution of clinically relevant small vessel vasculopathy. There is additional concern that a single measurement may not produce an accurate reflection of what is a heterogeneous disease.

To test the hypothesis that the occlusion technique is able to discriminate large vessel organised thrombus from distal vasculopathy, we performed occlusion pressures on patients with operable CTEPH, distal inoperable CTEPH and post-PEA residual CTEPH. In addition, we also undertook measurements in patients with idiopathic or connective tissue disease-associated pulmonary arterial hypertension (IPAH or CTD-PAH) as additional controls, where distal vasculopathy is traditionally accepted as predominant. In a select group of CTEPH patients we performed multiple measurements in different pulmonary segments to determine if there were differences within segments in the same patients.

\section{METHODS}

\section{Subjects}

59 subjects gave full written informed consent to these studies. The study was approved by the local research ethics committee (Huntington, UK) and conformed with the principles outlined in the Declaration of Helsinki. Subjects were diagnosed with CTEPH, IPAH or CTD-PAH according to standard classification $[6,7]$. The diagnosis and decision on operability was made by a multidisciplinary team of PEA surgeons, physicians and radiologists blinded to the results of the study using, additionally, a minimum two out of four imaging modalities; ventilation/ perfusion scanning, conventional pulmonary angiography, computed tomography pulmonary angiography (CTPA) and magnetic resonance imaging pulmonary angiography. Patients were deemed successfully operable if there was a technically good clearance of proximal disease and an intraoperative drop in mean pulmonary arterial pressure (mPAP). Subjects were routinely catheterised according to local protocol at 3 months post-PEA and therefore medium-term follow-up haemodynamics were available for analysis.

\section{Right heart catheterisation}

Right heart catheterisation was undertaken with the patient lying supine and breathing room air. Pulmonary haemodynamic measurements of $\mathrm{mPAP}$ and pulmonary arterial occlusion pressure (PAOP) were recorded using a 7-Fr Swan-Ganz catheter (Edwards Lifesciences, Newbury, UK) inserted via the right internal jugular vein. Cardiac output $(Q)$ was determined using the thermodilution method. Systemic arterial pressure and oxygen saturation $\left(\mathrm{S}_{\mathrm{a}}, \mathrm{O}_{2}\right)$ measurements were obtained noninvasively at $5 \mathrm{~min}$ intervals throughout the procedure. PVR was calculated using standard haemodynamic formulae.

\section{Occlusion pressure technique and analysis}

The catheter was positioned using fluoroscopic monitoring distally in a pulmonary artery. The wire-directed approach involved a wire being passed into an alternative segmental artery and subsequently being floated into a distal artery in the same manner as the standard flow-directed measurement. Therefore, although wire-directed these are still also flowdirected measurements in patent vessels of the same calibre. The vascular pressure signals were sampled at $200 \mathrm{~Hz}$ with the use of an analogue-to-digital converter (DAQCard-AI-16XE-50; National Instruments, Newbury, UK) and displayed and stored data encrypted on a personal computer. After single inflation of the pulmonary artery catheter, occlusion waveforms were recorded during breath-holding for $8 \mathrm{~s}$ at end-expiration. Measurements were performed in triplicate. In a subgroup of seven subjects with CTEPH, multiple sites were sampled by flow direction and additional catheter wire directed methods. A separate, blinded investigator performed a biexponential fitting of the pressure decay curve between the moment of occlusion and the PAOP, with normalisation to the MPAP. This has been previously described and is used here to derive occlusion pressure $\left(P_{\text {occl }}\right)[5,8]$. Rup (upstream pressure) was calculated as follows: Rup $\%=100 \times(\mathrm{mPAP}-$ Poccl $) /(\mathrm{mPAP}-\mathrm{PAOP})$.

\section{Statistics}

Relationships between variables and Rup values were analysed using linear regression and calculation of the Pearson correlation coefficient. Adherence to a Gaussian distribution was determined using a Kolmogorov-Smirnov method and between group differences were determined using a one-way ANOVA test. Results are presented as mean \pm SD unless otherwise stated. A receiver operating characteristic (ROC) curve was generated to establish a cut-off value for differentiating between operable and inoperable CTEPH. For all statistical tests a p-value $<0.05$ was considered significant.

\section{RESULTS \\ Subjects}

59 patients were sampled, with 47 patients completing the full protocol. In three subjects it was not possible to obtain an occlusion pressure, three were unable to breath-hold for the required time and six subjects had respiratory artefacts in their baseline occlusion pressure and, therefore, it was not possible to calculate their pressure decay accurately. Of these 12 subjects, eight were in the first 30 tested, with the remaining four in the last 29 and, therefore, there was a clear operator learning curve. Despite this the blinded observer ensured that only tracings of sufficient quality were analysed.

Of the 47 patients with analysable tracings, 14 had operable CTEPH, 15 had inoperable CTEPH, 13 had IPAH or CTP-PAH and five subjects had residual distal post-operative CTEPH (table 1). Of the operable patients, two died and therefore were not included in the successfully operable disease group. Both had significant distal disease on pre-operative assessment. The remainder of the operable group were surgically classified at the time of operation (using the THISTLETHWAITE et al. [9] classification) as predominantly type II/III disease and adjudged to have good technical clearance (table 1).

\section{In CTEPH Rup is not uniformly distributed across the pulmonary circulation}

Rup repeatability within single locations was good with a mean standard deviation of $3.4 \%$ and within-subject coefficient of variation of $4.4 \%$. In the seven patients with multiple lobe sampling there was wide variation (table 2). 


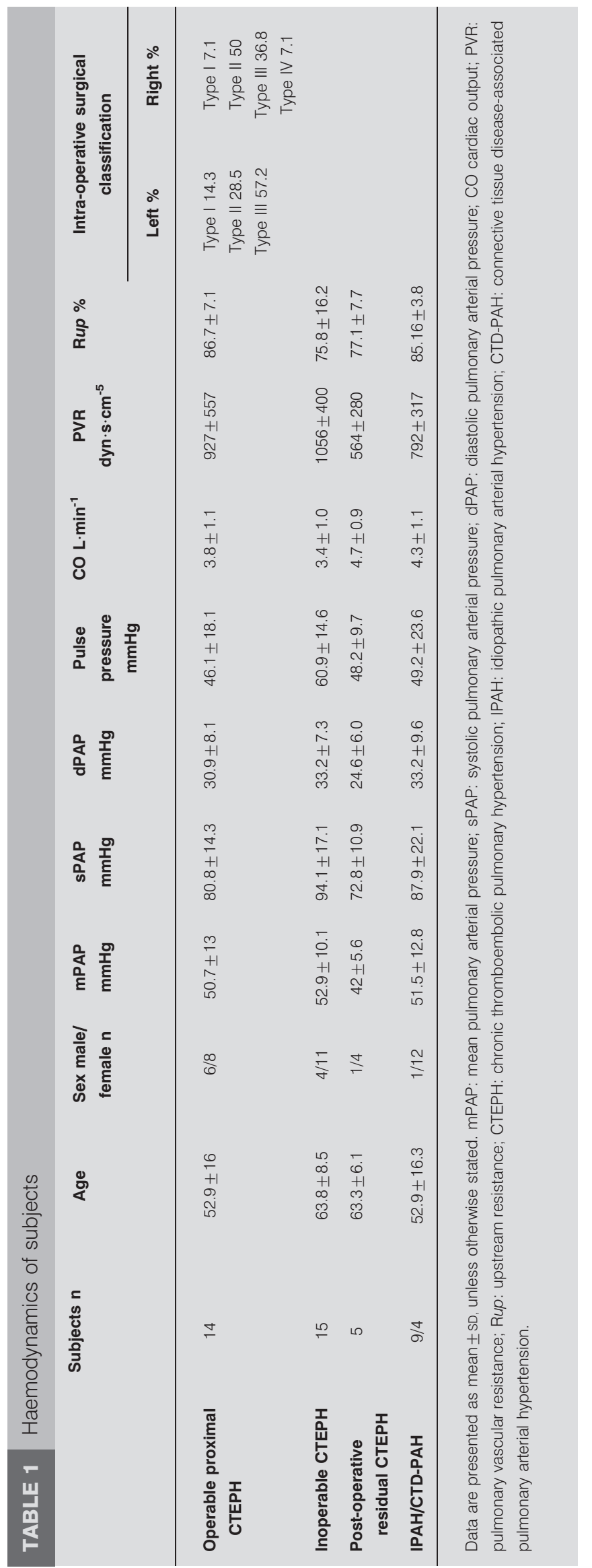

\section{Flow-directed Rup is significantly increased in proximal CTEPH}

There was a significant difference in Rup between the operable subjects and the two predominantly distal vasculopathic groups: operable CTEPH mean $87.3 \%$ (95\% CI 84.1-90.5\%); inoperable CTEPH mean 75.8\% (95\% CI 66.76-84.73\%) $\mathrm{p}=0.048$; IPAH/CTD-PAH mean 77.1\% (95\% CI 71.86-82.33\%) $\mathrm{p}=0.003$ (fig. 1). Looking at the sensitivity and specificity of the Rup in distinguishing between operable and inoperable disease, ROC curves demonstrate that Rup is capable of distinguishing between operable and inoperable disease with a significant area under the curve $(p=0.001)$ (fig. 1c). If we therefore assume that the purpose of the test is to identify inoperable disease, the cutoff point of 79.3 gives a $100 \%$ sensitivity (95\% CI 73.5-100\%) and specificity of $57.1 \%$ (95\% CI $28.9-82.3 \%)$. The optimum point for improving specificity on the ROC curve would be $83.8 \%$ which gives a sensitivity of $83.3 \%$ (95\% CI 51.6-97.9\%) and specificity of $71.4 \%$ (95\% CI $41.9-91.6 \%)$. If we adopt the more stringent definition of successful operative intervention of reduction to a PVR of $<300$ dyn $\cdot \mathrm{s}^{\cdot} \mathrm{cm}^{-5}$ there is no change to the results (fig. 1). In the operable group both patients who died were not included in the analysis as they could clearly not be classified as successfully operable disease but these two subjects had the lowest upstream pressures in the operative cohort $(68 \%$ and $73 \%$ ). One patient had significant post-operative pulmonary hypertension at 3 months despite a drop post-operatively, with a PVR of $1,040 \mathrm{dyn} \cdot \mathrm{s} \cdot \mathrm{cm}^{-5}$. Interestingly this patient had a flowdirected Rup of $96 \%$, but was also in the subgroup with multiple measurements and the wire-directed additional measurement was $49 \%$ (the lowest in the whole operative cohort). Postoperative mPAP did not correlate with Rup (fig. 2). Haemodynamic variables at 3 months also demonstrated no correlation to Rup, either using the flow-directed measurements or including the multiple sampling group (data not included), but our data was not statistically powered for this. Of interest, Rup did correlate moderately with PVR in the IPAH/CTD-PAH group $(\mathrm{r}=0.59, \mathrm{p}=0.03)$ (fig. 2).

\section{DISCUSSION}

There are a number of observations to be made from this work. From a technical viewpoint we have demonstrated that Rup measurements are reliably repeatable, although there are a significant proportion of subjects in whom they are not obtainable. It is likely that there is an operator learning curve, as demonstrated by the higher success rates towards the end of the study. There was significant heterogeneity when contralateral lung multiple wire-directed sites were sampled and compared to the flow-directed measurements. It is possible that this represents the technical differences between a flow-directed measurement and a wire-directed measurement, which one might expect to end in a less well-perfused segment. This does fit well with our understanding of the differences between CTEPH and IPAH, and in particular the heterogeneity of disease distribution. Perhaps more surprising is the demonstration that although there are statistically significant differences in partitioned small vessel resistance between disease categories, there does appear to be a spectrum of resistance across all of the disease groups. This may have importance in understanding pathophysiology and response to treatment. 


\section{TABLE 2 Subjects with multiple sampling}

\begin{tabular}{|c|c|c|c|c|c|}
\hline Subject & Diagnosis & Pre-operative PVR dyn $\cdot \mathbf{s} \cdot \mathrm{cm}^{-5}$ & Flow-guided Rup \% & Wire-directed Rup \% & $\begin{array}{c}\text { Post-operative 3-month PVR } \\
\text { dyn } \cdot \mathbf{s} \cdot \mathrm{cm}^{-5}\end{array}$ \\
\hline 2 & Operable CTEPH & 416 & 96 & 49 & 1040 \\
\hline 3 & Operable CTEPH & 800 & 94.5 & 71.5 & 424 \\
\hline 4 & Operable CTEPH & 2216 & 73 & 90.8 & Died \\
\hline 7 & Inoperable CTEPH & 1440 & 78 & 67 & Not applicable \\
\hline
\end{tabular}

PVR: pulmonary vascular resistance; Rup: upstream resistance; CTEPH: chronic thromboembolic pulmonary hypertension.

Flow-directed Rups are higher in operable predominantly proximal CTEPH than in inoperable CTEPH and IPAH/CTD. For the single measurement technique, ROC curves demonstrate good sensitivity but lower specificity for distinguishing between operable and inoperable disease. One explanation for this is the heterogeneous nature of disease, which means that
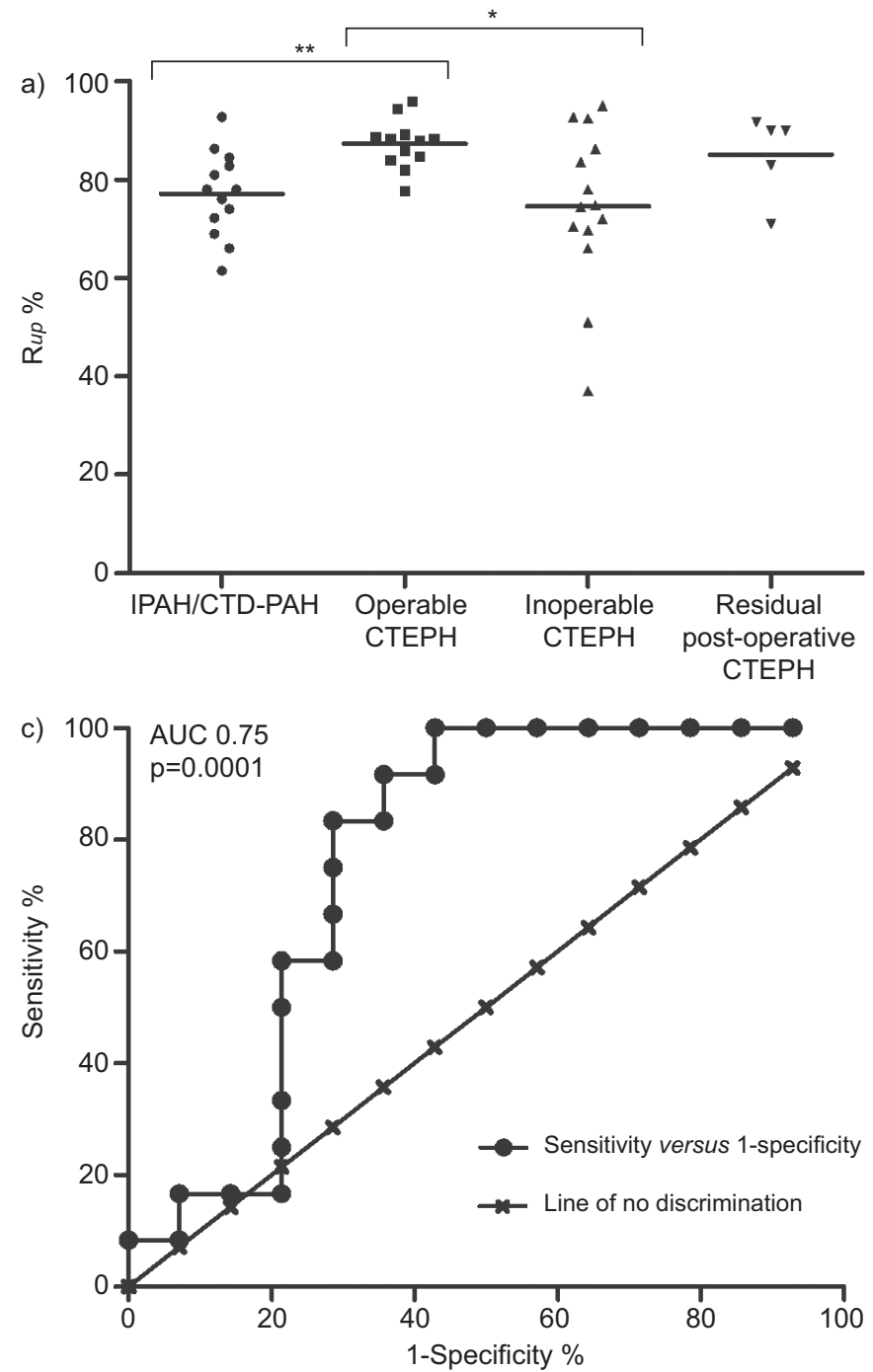

distal vasculopathy can be missed by a single measurement. Another explanation is that the technique partitions at a vessel size that misses significant downstream resistance. The occlusion technique is suggested to estimate pressure in vessels above $100-150 \mu \mathrm{m}$ (with the rest being made up of vessels below $150 \mu \mathrm{m}$, capillaries and veins) [10]. Anatomical studies show

b)

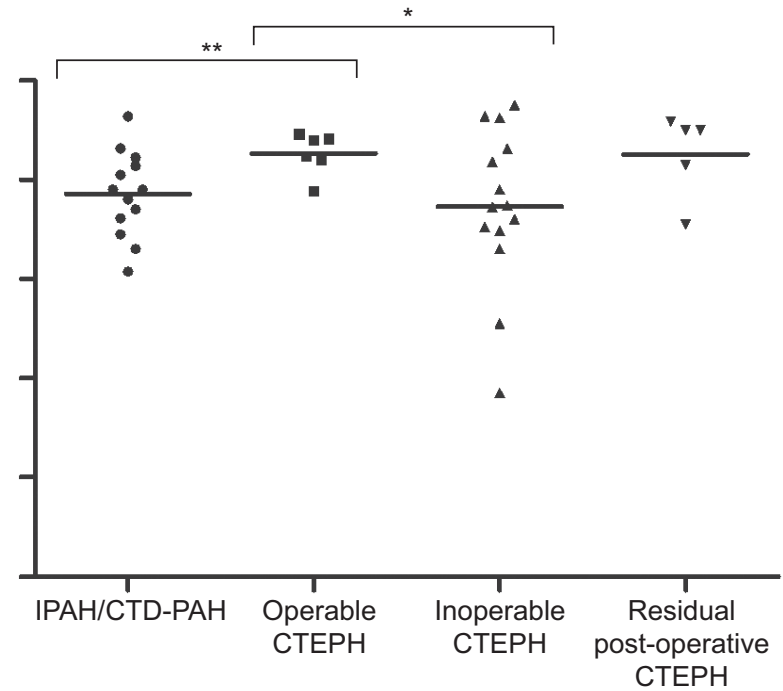

FIGURE 1. Upstream resistance (Rup) in pulmonary hypertension. a) Rup in all groups; b) Rup if cut-off for successfully operable disease is set as a 3-month pulmonary vascular resistance $<300$ dyn $\cdot \mathrm{s} \cdot \mathrm{cm}^{-5}$; c) receiver operating characteristic curves for sensitivity/ specificity of the Rup in distinguishing operable from inoperable chronic thromboembolic pulmonary hypertension (CTEPH). IPAH/ CTD-PAH: idiopathic pulmonary arterial hypertension/connective tissue disease-related pulmonary arterial hypertension; AUC: area under the curve; ${ }^{*}: p<0.05 ; * *: p<0.01$. 

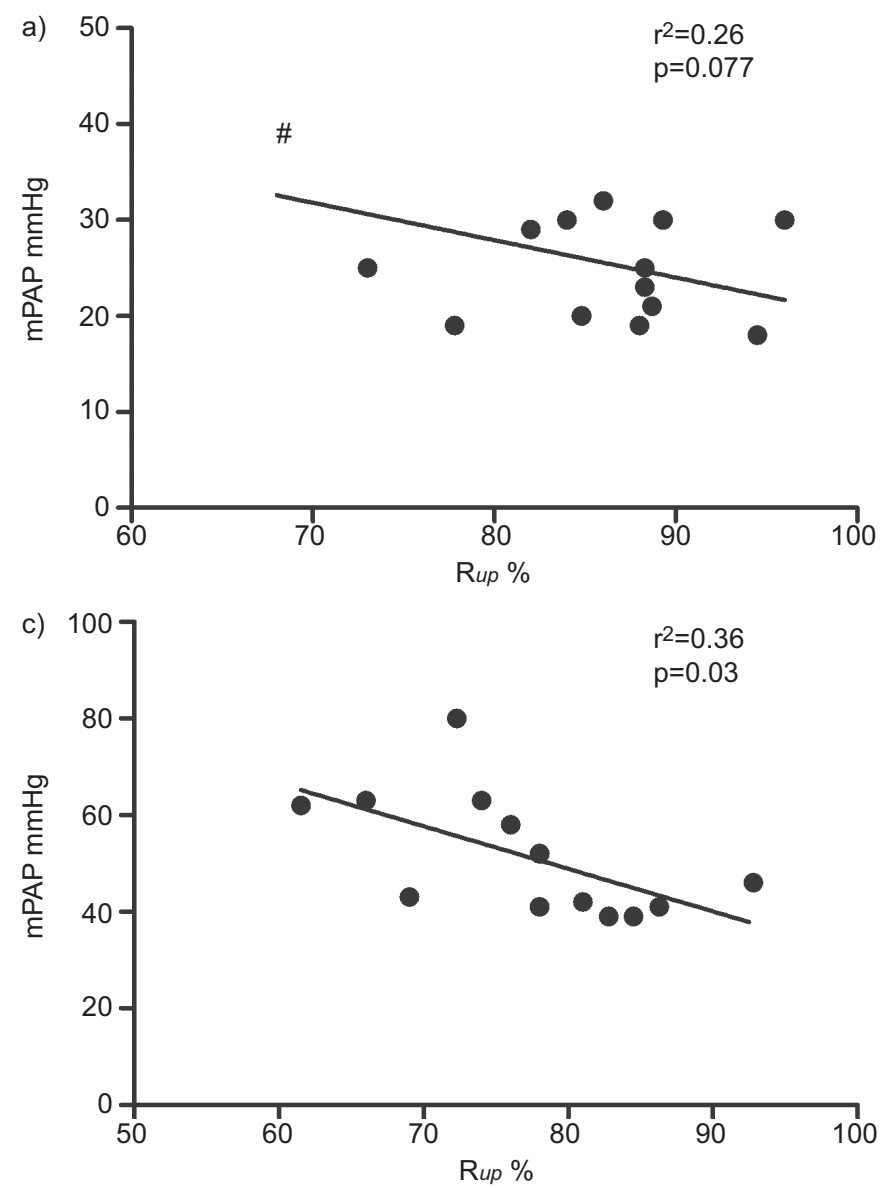

b)

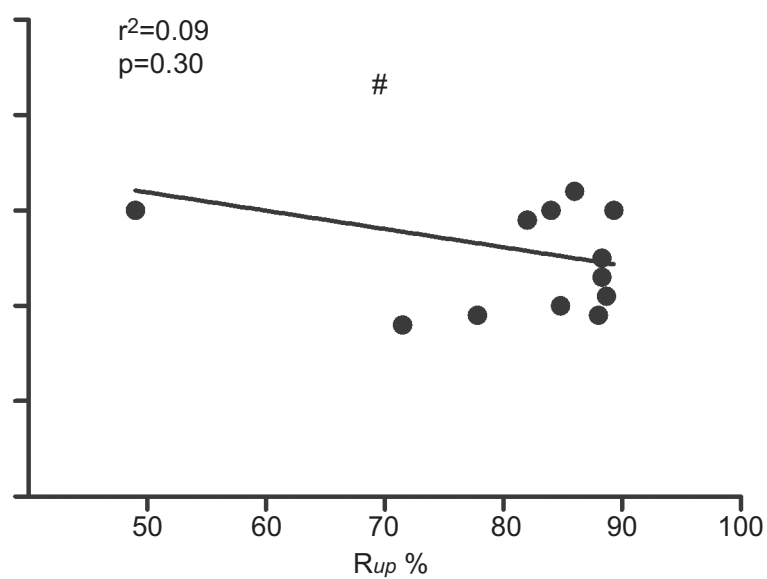

FIGURE 2. Correlations between upstream resistance (Rup) and immediate post-operative mean pulmonary arterial pressure (mPAP) in operable chronic thromboembolic pulmonary hypertension (CTEPH) and idiopathic pulmonary arterial hypertension/connective tissue diseaserelated pulmonary arterial hypertension (IPAH/CTD-PAH). a) Correlation in operable cohort with flow-directed occlusion based measurements; b) correlation in operable cohort using the lowest measurement including flow-directed and wire-directed occlusion based measurements; c) correlation in IPAH and CTD-PAH. \#: subject died in post-operative period. that in humans the muscularised small arteries are to be found in vessels larger than $100 \mu \mathrm{m}$ [11]. These small muscularised arteries have long been held to be the major contributors to resistance in PAH [12]. It is therefore possible that the occlusion technique does not interrogate the correct range of vessel calibre, or in other words is mislabelling a significant portion of resistance in these small vessels as upstream. If this is the case, it is possible that by changing the analysis of the decay curve we can capture more clinically relevant resistance in vessels larger than $100-150 \mu \mathrm{m}$. The mislabelled resistance hypothesis is supported by the fact that the IPAH/CTD-PAH and distal CTEPH cohorts had a much higher Rup than would be expected if the resistance had been accurately partitioned into clinically relevant small and large vessels. Despite this there was a moderate correlation between Rup and mPAP in IPAH/CTD$\mathrm{PAH}$. In other words, as would be expected in distal predominant pathology, as the distal component of resistance increases so does the pulmonary artery pressure. Therefore, although Rup partitioning may miss a component of downstream resistance, it still increases relative to traditional haemodynamic parameters. The occlusion pressure technique is currently the only technique able to compartmentalise resistance in vivo.

In our studies a low single flow-directed Rup was a risk factor for operative mortality. Previously a flow-directed Rup of $<60 \%$ was demonstrated to be a significant risk factor for mortality [5]. Both of the subjects who died in our cohort had a higher threshold than this (at 68\% and 73\%). The flow-directed Rup did not pick up the patients with significant post-operative pulmonary hypertension. Although multiple sampling was not undertaken in all of the CTEPH subjects, if the multiple wire-directed values are included, the subject with the highest post-operative PVR also had the lowest Rup in the operable cohort (49\%). This subject had been identified as high-risk pre-operatively with possible mixed proximal/distal disease and a PVR before targeted therapy of $1,040 \mathrm{dyn} \cdot \mathrm{s} \cdot \mathrm{cm}^{-5}$. There was a post-operative fall in $\bar{P}_{\text {pa }}$ and PVR and CTPA at 3 months confirmed clearance, but with some residual distal disease. Despite this, post-operatively at 3 months, targeted therapy had to be restarted after demonstration of significant residual pulmonary hypertension that had increased after withdrawal of bosentan. In addition the other subject in the multiple sampling group with a PVR $>300$ dyn. $\mathrm{s} \cdot \mathrm{cm}^{-5}$ at 3 months (424 dyn $\cdot \mathrm{s} \cdot \mathrm{cm}^{-5}$ ) had a wire-directed Rup of $71.5 \%$. Because multiple sampling was only obtained in a subgroup we cannot comment on its comparative benefit in the study. When multiple sites were sampled there was heterogeneity within different lobes. The heterogeneity of disease may mean it is possible to miss significant distal disease with one measurement, but the presence of a low Rup is again confirmed to be an indicator of distal vasculopathy.

A potential criticism of this work is the definition of operable CTEPH. This is a difficult area as there is no gold standard definition of success. Additionally, thresholds for surgical operability differ worldwide, mainly dependent on surgical experience. In our study the subjects were carefully diagnosed in 
a multidisciplinary meeting with three or four imaging modalities. We present in our analysis all the patients who survived the operation despite six subjects having PVR $>300 \mathrm{dyn} \cdot \mathrm{s}^{\cdot} \mathrm{cm}^{-5}$. All of these patients were adjudged to have a good technical clearance by the attending surgeon and on subsequent imaging, with an immediate post-operative reduction in PVR and improvement in 6-min walk. Regardless, if more stringent criteria are adopted (a post-operative PVR $<300 \mathrm{dyn} \cdot \mathrm{s} \cdot \mathrm{cm}^{-5}$ ) the same results are generated. Additionally, it is possible that distal inoperable disease has been misclassified. To address this we performed a post hoc analysis case review to exclude anyone with any doubt about the clarity of the diagnosis of operable CTEPH versus inoperable (see online supplementary figure S1) but again this did not significantly alter our results. There remains the possibility that in the subjects diagnosed as inoperable, there are proximal lesions contributing to vascular resistance. It is reassuring in this regard that our current screening criteria are confirmed by Rup analysis to select out patients with significant distal resistance. To mitigate this criticism we have included subjects with IPAH, CTD-PAH and residual post-operative CTEPH. In these subjects, who have distal pathology, we see the same statistically significant difference in mean Rup but with a higher than expected proportion of upstream resistance.

In conclusion, we have found that Rup as measured by the occlusion technique is increased in operable predominantly proximal CTEPH when compared with inoperable CTEPH and IPAH/CTD-PAH. This technique is confirmed as reliable and repeatable and can be performed during standard right heart catheterisation. Given that all patients undergoing assessment for PEA have a right heart catheter as part of their workup, it would not be technically difficult or expensive to perform the analysis, although there is a demonstrable operator learning curve. As yet our data does not support the clinical use of this technique in routine assessment. If further work is to be done, thought needs to be given to the analysis of the decay curve, and wire-directed measurement may provide additional information on disease heterogeneity in CTEPH.

\section{SUPPORT STATEMENT}

This research was supported by the National Institute for Health Research (NIHR) Cambridge Biomedical Research Centre.

\section{STATEMENT OF INTEREST}

A statement of interest for P. Fesler can be found at www.erj. ersjournals.com/site/misc/statements.xhtml

\section{REFERENCES}

1 D'Armini AM, Cattadori B, Monterosso C, et al. Pulmonary thromboendarterectomy in patients with chronic thromboembolic pulmonary hypertension: hemodynamic characteristics and changes. Eur J Cardiothorac Surg 2000; 18: 696-701.

2 Masuda M, Nakajima N. Our experience of surgical treatment for chronic pulmonary thromboembolism. Ann Thorac Cardiovasc Surg 2001; 7: 261-265.

3 Tscholl D, Langer F, Wendler O, et al. Pulmonary thromboendarterectomy - risk factors for early survival and hemodynamic improvement. Eur J Cardiothorac Surg 2001; 19: 771-776.

4 Thistlethwaite PA, Kemp A, Du L, et al. Outcomes of pulmonary endarterectomy for treatment of extreme thromboembolic pulmonary hypertension. J Thorac Cardiovasc Surg 2006; 131: 307-313.

5 Kim NH, Fesler P, Channick RN, et al. Preoperative partitioning of pulmonary vascular resistance correlates with early outcome after thromboendarterectomy for chronic thromboembolic pulmonary hypertension. Circulation 2004; 109: 18-22.

6 Klepetko W, Mayer E, Sandoval J, et al. Interventional and surgical modalities of treatment for pulmonary arterial hypertension. J Am Coll Cardiol 2004; 43: 73S-80S.

7 Simonneau G, Robbins IM, Beghetti M, et al. Updated clinical classification of pulmonary hypertension. J Am Coll Cardiol 2009; 54: S43-S54.

8 Fesler P, Pagnamenta A, Vachiéry JL, et al. Single arterial occlusion to locate resistance in patients with pulmonary hypertension. Eur Respir J 2003; 21: 31-36.

9 Thistlethwaite PA, Mo M, Madani MM, et al. Operative classification of thromboembolic disease determines outcome after pulmonary endarterectomy. J Thorac Cardiovasc Surg 2002; 124: 1203-1211.

10 Kafi SA, Melot C, Vachiery JL, et al. Partitioning of pulmonary vascular resistance in primary pulmonary hypertension. J Am Coll Cardiol 1998; 31: 1372-1376.

11 Harris $P$, Heath D. The Human Pulmonary Circulation. Edinburgh, Livingstone, 1962; pp. 62-63.

12 Wagenvoort CA, Wagenvoort N. Primary pulmonary hypertension: a pathologic study of the lung vessels in 156 clinically diagnosed cases. Circulation 1970; 42: 1163-1184. 